\title{
Clathrate Hydrate Formation and Micellization of Tetrabutylammonium 10-Undecenoate
}

\author{
Clifford E. Larrabee Jr.1, Mary R. Warmin'1, Melissa J. Howard ${ }^{2}$ \\ ${ }^{1}$ University of Cincinnati Clermont College \\ Batavia, Ohio, USA \\ cliff.larrabee@uc.edu; warminmr@ucmail.uc.edu \\ 2University of Cincinnati, College of Engineering \\ Cincinnati, Ohio, USA \\ howardmj@mail.uc.edu
}

\begin{abstract}
The hydrophobic effect can give rise to both clathrate hydrate formation and micellization, depending on the molecular composition of the solute, temperature, and pressure. However, only a single compound, tetrabutylammonium dodecanoate, TBAD, has been shown to induce both types of self-assembly. Here we examine a second clathrate-forming surfactant. Tetrabutylammonium 10undecenoate, TBAU, is shown to form a clathrate with a hydration number of 22.6 and a melting point of $293.32 \pm 0.16$ $K$, and also to form micelles with a critical micelle concentration, CMC, of $0.032 \pm 0.004 \mathrm{~mol} \mathrm{dm}^{-3}$ at $298.2 \pm 0.3$ K. Solubilization of a hydrophobic dye, Oil Blue N, by TBAU was compared to a series of tetra-n-alkylammonium 10undecenoates at $296 \pm 2 \mathrm{~K}$. The CMC decreased and the solubilization power of the surfactant increased with the number of carbons in the counterion. No special, clathrate hydrate forming effects were evident above the clathrate hydrate melting point.
\end{abstract}

Keywords: Clathrate, Micelle, Hydrophobic effect, Solubilization, Model system, Drug delivery.

(C) Copyright 2014 Authors - This is an Open Access article published under the Creative Commons Attribution License terms (http://creativecommons.org/licenses/by/3.0).

Unrestricted use, distribution, and reproduction in any medium are permitted, provided the original work is properly cited.

\section{Introduction}

Clathrate hydrates are supramolecular frameworks composed of polyhedral water cages supported by relatively small guest molecules. Fully hydrogen-bonded water molecules form the sides of the polyhedral cages with diameters of approximately one nanometer in a cubic or hexagonal crystal system. [1] Gas hydrates are currently a major area of research in the fuel industry and also have been considered for advanced drug delivery applications. [2]

Micelles are nano-sized, self-assembled aggregates of surfactants in a water medium. [3], [4] Because surfactants are amphiphilic, they associate with their hydrophobic tails in the interior of the aggregate, away from the water, and with their hydrophilic headgroups in the outer regions of the aggregate, in contact with the water. The hydrophobic region in the interior of the micelle provides a favorable environment for solubilization of hydrophobic substrates. Historically, their primary application has been in soaps and detergency, but they are increasingly important in drug delivery systems. [6], [7] A surfactant that could localize guest molecules, either by clathrate formation or by micellar solubilization, might provide a simple model for multifunctional nanoparticles for drug delivery systems. [8]

The number of surfactant compounds known to form clathrate hydrates is small. [9], [10] Of the known clathrate-forming surfactants, only one, tetrabutylammonium dodecanoate, TBAD, has also been shown to form micelles in aqueous solution. [11], [12] Zana [11] reported an unexpectedly small micelle aggregation number for TBAD at $298 \mathrm{~K}$, but little else is known about any unusual properties of these compounds.

The overall goal of this research is to develop a model system for multifunctional, nanosized media 
with constrained geometries. [13] The 10-undecenoate anion was chosen because it is polymerizable [14] and its micellization properties are well characterized [15]. The tetrabutylammonium cation was chosen because it is a well-known clathrate former [16] that has been shown to form clathrate hydrates with long-chain carboxylates [9], [10]. Oil Blue $\mathrm{N}$ was chosen because of the resemblance of its molecular structure to that of Mitoxantrone (Figure 1), which is used to treat leukemia and prostate cancer.

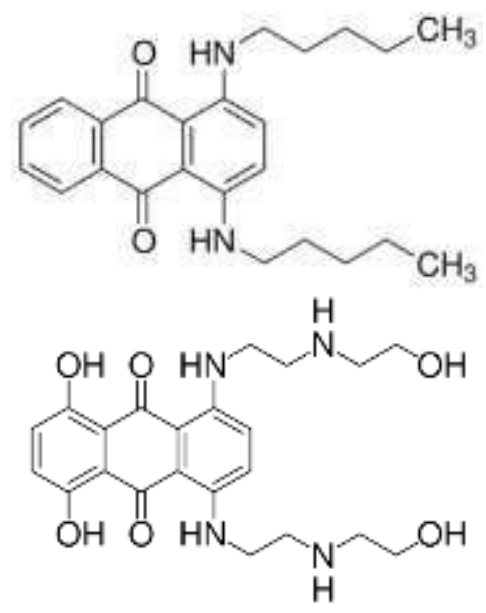

Figure 1. Molecular structures of Oil Blue N (above) and Mitoxantrone (below)

The specific objectives of the project described here were to establish that tetrabutylammonium 10undecenoate, TBAU, forms both stable clathrates and micelles, and to investigate its solubilization of a hydrophobic dye at temperatures above the clathrate melting point.

\section{Materials and Methods}

All solutions were made up with ACS Reagent Grade Type I water (Ricca Chemical Company). Tetrabutylammonium fluoride, TBAF, solutions were prepared from TBAF $3 \mathrm{H}_{2} \mathrm{O}$ (Acros, 99\%). The 10undecenoate salt solutions were prepared by neutralization of 10-undecenoic acid (Acros, 99\%) with the appropriate base after standardizing the base with potassium hydrogen phthalate (Fisher, ACS). The bases were tetramethylammonium hydroxide (Acros, 25\% in water), tetraethylammonium hydroxide (Acros, $25 \%$ in water), tetrapropylammonium hydroxide (Acros, 25\% in water), and tetrabutylammonium hydroxide (Acros, $40 \%$ in water).

\subsection{Hydrate Melting Point and Morphology}

Melting points and photomicrographs were taken with a polarizing microscope using a custom thermal stage controlled by a Lauda RC 6 circulator. Two thermistors, attached to the microscope slide, were calibrated with a NIST-traceable thermometer. Temperature stability was found to be $\pm 0.11 \mathrm{~K}$ and the average difference in temperature between the top and bottom of the slide was $0.05 \mathrm{~K}$ or less. Total random error is estimated to be $\pm 0.16 \mathrm{~K}$. Accuracy was determined by comparing the observed melting point of gallium (Acros, 99.9999\%), $302.90 \mathrm{~K}$, to the accepted value, $302.91 \mathrm{~K}$ [17]. At this temperature, any systematic error is considerably less than the random error.

Solutions of known composition were placed in a microscope well slide, covered with a glass cover slip, and sealed. Each slide was placed in the constanttemperature stage at about $278 \mathrm{~K}$ until crystals formed. At this point, the temperature was raised slowly, with repeated recycling through melting and refreezing of the sample. Photomicrographs were taken during the recycling process and the melting points were taken as the highest temperature where crystals were still present.

\subsection{Conductivity}

The critical micelle concentration, CMC, of tetrabutylammonium 10-undecenoate was determined by the conductivity method described by Sprague, et al. [18]. Conductance was measured at $298.2 \pm 0.3 \mathrm{~K}$ using an Amber Science Model 1056 conductivity meter and P/N 515 cell.

\subsection{Dye Solubilization}

Solutions were prepared by adding excess Oil Blue $\mathrm{N}$ (MP Biomedicals) to the surfactant solutions, sonicating for 30 minutes, allowing to settle overnight, and filtering (Millipore PVDF $0.45 \mu \mathrm{m}$ ) into glass cuvettes. Absorbance measurements were made at 600 $\mathrm{nm}$ with an Ocean Optics USB4000 UV/Vis spectrometer. The molar extinction coefficient of Oil Blue $\mathrm{N}$ at this wavelength was determined in ethanol to be $4.2 \pm 0.1 \times 10^{3} \mathrm{~m}^{2} \mathrm{~mol}^{-1}$. All measurements were made at room temperature, $296 \pm 2 \mathrm{~K}$.

Solubilization data were analysed following the general procedures described by Tehrani-Bagha and Holmberg [19] as modified below for the essentially water-insoluble Oil Blue N. 
Assuming a water insoluble dye, the molar solubilization capacity or solubilization power, SP, of a surfactant is given by equation (1)

$$
S P=c_{D} /\left(c_{S}-C M C\right)
$$

where $c_{\mathrm{D}}$ is the concentration of the dye in the surfactant solution and $c_{\mathrm{S}}$ is the concentration of the surfactant. Equation (1) can be rearranged to equation (2)

$$
c_{D}=S P \times c_{S}-S P \times C M C
$$

A plot of $c_{\mathrm{D}}$ vs. $c_{\mathrm{S}}$ gives a straight line with a slope equal to SP and an $\mathrm{x}$-intercept equal to the CMC.

For the regression analysis of the solubility data, equation (1) was rewritten as equation (3) in order to separate the fitting parameters and better estimate their uncertainties.

$$
c_{S}=\frac{1}{S P} \times c_{D}+C M C
$$

\section{Results}

\subsection{Clathrate Formation}

Two aqueous solutions of tetrabutylammonium fluoride, TBAF, were prepared. The first, 30.68\% TBAF, corresponds to a hydration number of 29.7 for the crystal. This hydrate had a melting point of $300.85 \pm 0.16 \mathrm{~K}$ and formed polyhedral crystals shown on the left in figure 2. The second, 32.83\% TBAF, corresponds to a hydration number of 32.8. This hydrate had a melting point of $300.34 \pm 0.16 \mathrm{~K}$ and formed elongated prisms shown on the right in Figure 2.
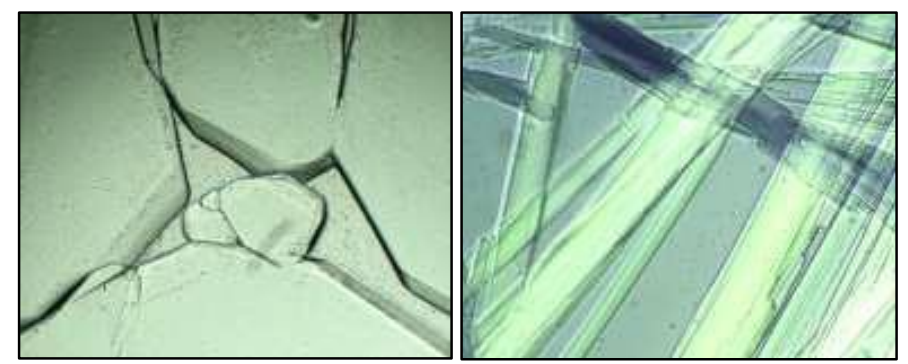

Figure 2. Polyhedral crystals of TBAF-29.7H2O crystals (left) and elongated prisms of TBAF $32.8 \mathrm{H} 2 \mathrm{O}$ (right). The fields are $300 \times 375 \mu \mathrm{m}$ (left) and $850 \times 1050 \mu \mathrm{m}$ (right)

Several aqueous solutions of TBAU were prepared. The hydrate with the highest melting point, $293.32 \mathrm{~K}$, was formed from a $51.11 \%$ solution, corresponding to a hydration number of 22.6. A representative sample of the polyhedral crystals formed from this solution is shown in Figure 3.

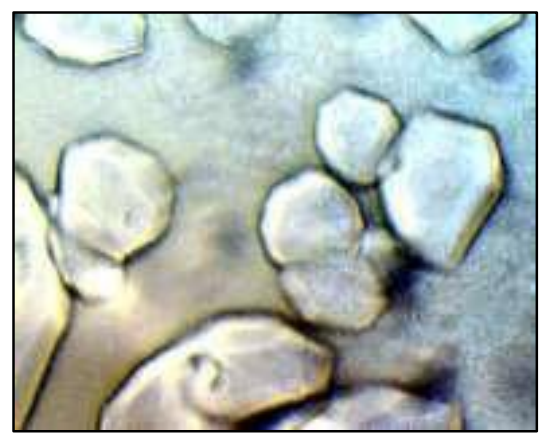

Figure 3. Polyhedral crystals of TBAU $22.6 \mathrm{H}_{2} \mathrm{O}$. The field is $300 \times 375 \mu \mathrm{m}$

\subsection{Micelle Formation}

Conductivity results are summarized in Figure 4. The lines on the graph are linear-regression fits to the data. For the lower concentration points, the $y$ intercept was set to zero. Points within $\pm 10 \%$ of the CMC were excluded from the regression analysis. Correlation coefficients for both lines were better than 0.99. The CMC, determined from the intersection of the two lines was $0.032 \mathrm{~mol} \mathrm{dm}^{-3}$ with a standard error of $0.004 \mathrm{~mol} \mathrm{dm}^{-3}$.

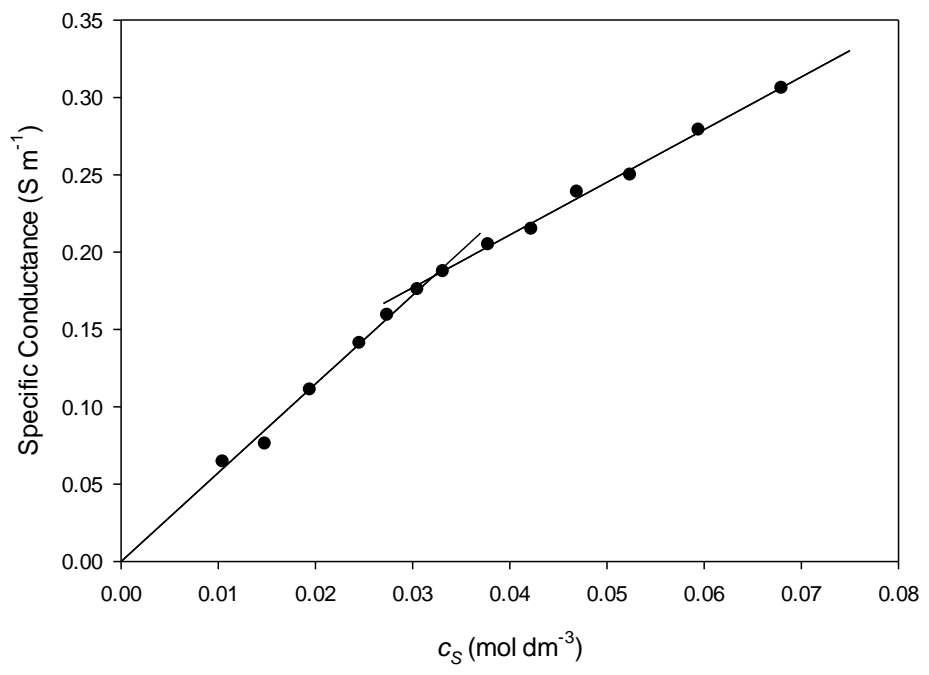

Figure 4. TBAU specific conductance as a function of surfactant concentration

\subsection{Solubilization}

Solubilization measurements of Oil Blue $\mathrm{N}$ by tetramethylammonium 10-undecenoate, TMAU, tetraethylammonium 10-undecenoate, TEAU, and tetrapropylammonium 10 -undecenoate, TPAU, as well 
as TBAU, were made at $296 \pm 2 \mathrm{~K}$. The results are summarized in Figure 5 and Table 1 . The quality of the linear fits was generally good, correlation coefficients ranged from 0.92 for TPAU to 0.99 for TMAU. The CMC for TBAU determined by this method, $0.031 \pm 0.003$ mol dm${ }^{-3}$, is indistinguishable from the conductivity value $(\mathrm{t}=0.63, \mathrm{p}=0.54)$.

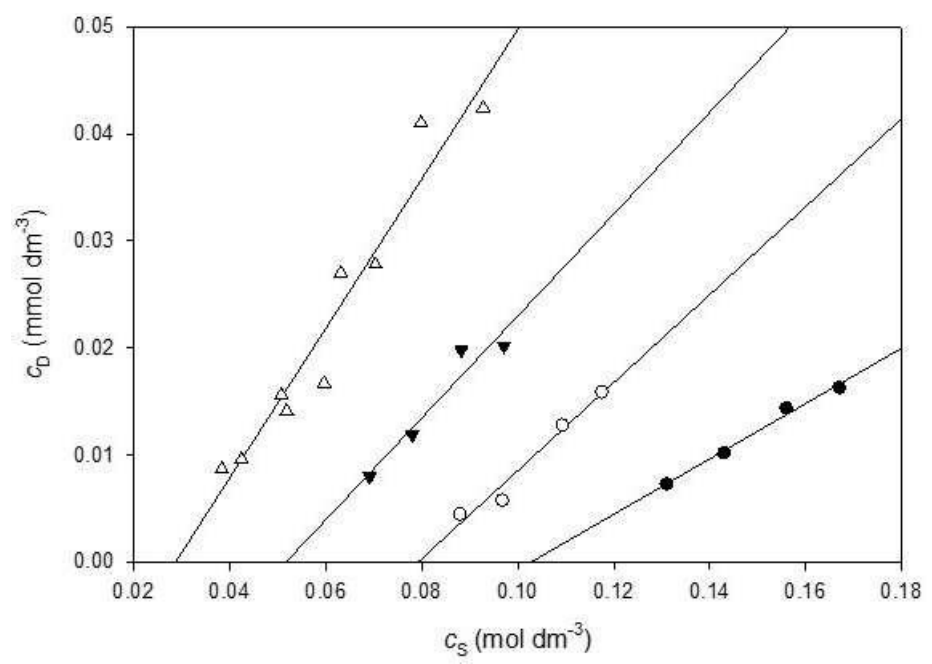

Figure 5. Dye solubilization as a function of surfactant concentration. $\triangle$ TBAU, $\nabla$ TPAU, o TEAU, and $\bullet$ TMAU

Table 1. Critical Micelle Concentrations and Solubilization Power

\begin{tabular}{|c|c|c|c|c|}
\hline & $\begin{array}{c}\text { CMC } \\
\left(\mathrm{mol} \mathrm{dm}^{-}\right.\end{array}$ & $\begin{array}{c}\text { Std. } \\
\text { Error }\end{array}$ & SP $\times 10^{3}$ & Std. Error \\
\hline TMAU & 0.103 & 0.003 & 0.26 & 0.02 \\
\hline TEAU & 0.080 & 0.003 & 0.43 & 0.06 \\
\hline TPAU & 0.054 & 0.006 & 0.52 & 0.11 \\
\hline TBAU & 0.031 & 0.003 & 0.74 & 0.07 \\
\hline
\end{tabular}

\section{Discussion}

\subsection{Clathrate Formation}

The results for TBAF are in excellent agreement with those of Rodionova, et al. [16]. Two distinct hydrates were formed with hydration numbers of 29.7 and 32.8. Their crystals were polyhedral and elongated prisms, respectively, and their melting points were $300.85 \pm 0.16 \mathrm{~K}$ and $300.34 \pm 0.16 \mathrm{~K}$, respectively.

The observed melting point of TBAU $22.6 \mathrm{H} 2 \mathrm{O}$, $293.32 \pm 0.16 \mathrm{~K}$, is close to the melting point of its saturated analog, tetrabutylammonium undecanoate, 293.7 K [9]. The polyhedral crystals, Figure 3, are similar to those of TBAF, Figure 2. The x-ray crystal structure of TBAF-29.7 has been determined; the water framework is isostructural with the cubic structure-I of gas hydrate clathrates. [16]

Considering the above results, we conclude that TBAU $22.6 \mathrm{H} 20$ almost certainly has a clathrate-like structure. It should be noted that TBAF hydrates are sometimes referred to as semi-clathrate hydrates because the fluoride ion replaces one of the water molecules in the hydrate framework. [20] Also, the hydrate crystal structure of TBAU is undoubtedly more complex than that for TBAF since the 10undecenoate anion is too large to simply replace a water molecule in the water lattice.

\subsection{Micelle Formation}

The conductivity of TBAU in water at $298.2 \mathrm{~K}$ shows clear evidence of micelle formation, with a welldefined break in the specific conductance vs. concentration plot indicating a CMC of $0.032 \pm 0.004$ mol dm${ }^{-3}$. This is much lower than the value of 0.117 $\pm 0.001 \mathrm{~mol} \mathrm{~kg}^{-1}$ reported earlier for sodium 10undecenoate. [18] The tetrabutylammonium counterion shows a very strong effect, lowering the onset of micellization by a factor of more than three.

\subsection{Solubilization}

The solubilization experiments with the series of tetra- $n$-alkylammonium 10 -undecenoates were designed primarily to see if the clathrate-forming capacity of TBAU would have any influence on solubilization at temperatures above the clathrate melting point. The solubilization power of TBAU was clearly the strongest of the group. However, there appeared to be regular and predictable trends in both CMCs and SPs with the number of carbon atoms in the counterions.

There is an empirical relationship, first noted by Klevens [21], between the logarithm of the CMC and number of carbons in the surfactant tail. The same effect was found by Wang, et al. [22] for the number of carbons in the counterions TMA, TEA, TPA, and TBA for perfluorooctanoates and can be seen in the CMCs of TMA, TEA, TPA, and TBA dodecyl sulfates. [23] For TMAU, TEAU, TPAU, and TBAU, the relationship is shown in Figure 6. The linear fit is acceptable ( $\mathrm{F}=0.95$, $p=0.46)$ and there is no evidence of exceptional behaviour for the clathrate-forming TBAU. 


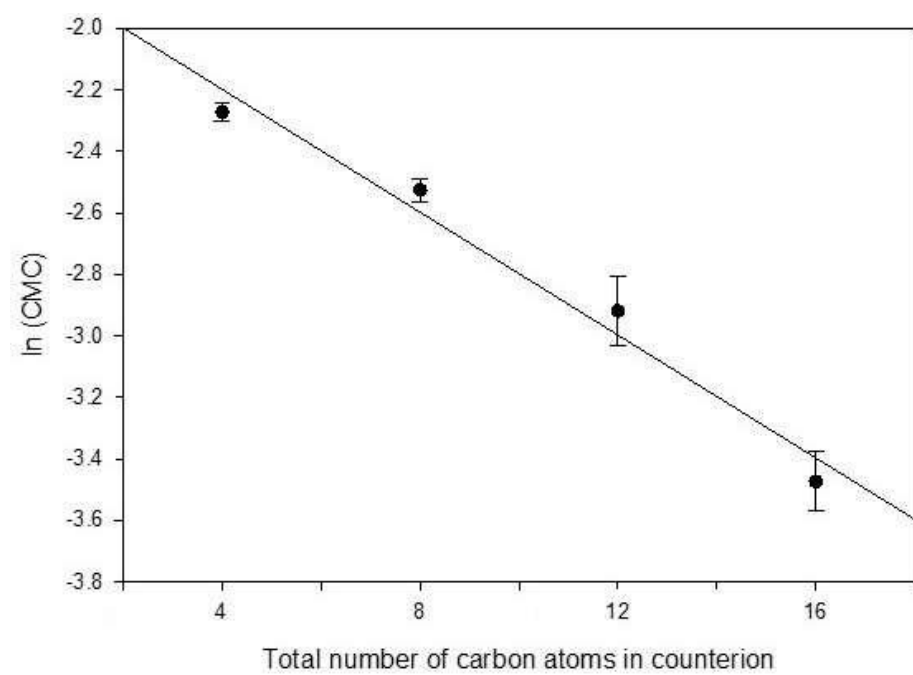

Figure 6. In (CMC) as a function of the total number of carbons in the counterion

The logarithm of solubilization power was also plotted as a function of total carbons for the four counterions, Figure 7, and a satisfactory linear fit was found $(F=0.21, p=0.82)$. This supports the hypothesis that at $296 \mathrm{~K}$, there is no special effect due to the potential for clathrate formation by TBAU. The relative effectiveness of TBAU in solubilizing Oil Blue $\mathrm{N}$ is attributed to increasing hydrophobicity of the counterion with increasing number of carbon atoms.

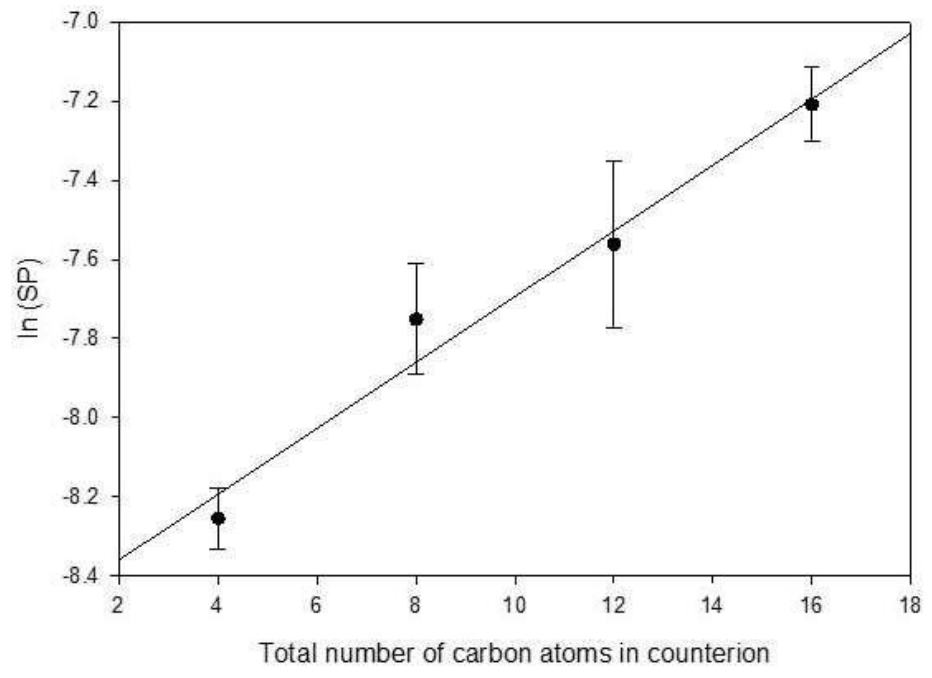

Figure 7. In (SP) as a function of the total number of carbons in the counterion

\section{Conclusion}

Tetrabutylammonium 10-undecenoate forms a stable hydrate with a hydration number of 22.6 and a melting point of $293.32 \pm 0.16 \mathrm{~K}$. It almost certainly has a clathrate-like crystal structure.

Tetrabutylammonium 10-undecenoate forms micelles in water at a CMC of $0.032 \pm 0.004 \mathrm{~mol} \mathrm{dm}^{-3}$. It is effective at solubilizing Oil Blue $\mathrm{N}$, a hydrophobic dye.

In the series of tetra- $n$-alkylammonium 10undecenoates, the relatively low CMC and relatively high SP of TBAU, can be attributed to increasing hydrophobicity of the counterion due to the increasing number of carbon atoms. No extraordinary clathrate hydrate forming effects are found above the hydrate melting point.

\section{References}

[1] J.W. Steed, D.R. Turner, K.J. Wallace, Core Concepts in Supramolecular Chemistry and Nanochemistry, West Sussex, England: Wiley, 2007.

[2] N. Blagden, M. de Matas, P.T. Gavan, and P. York, "Crystal Engineering of Active Pharmaceutical Ingredients to Improve Solubility and Dissolution Rates," Advanced Drug Delivery Reviews, vol. 59, pp. 617-630, 2007.

[3] J.N. Israelachvili, D.J. Mitchell, and B.W. Ninham, "Theory of Self-Assembly of Hydrocarbon Amphiphiles into Micelles and Bilayers," Journal of the Chemical Society, Faraday Transactions 2: Molecular and Chemical Physics, vol. 72, pp. 15251568, 1976.

[4] D. Chandler, "Interfaces and the Driving Force of Hydrophobic Assembly," Nature, vol. 437, pp. 640646, 2005.

[5] C.O. Rangel-Yagui, A. Pessoa Jr., and L.C. Taveres, "Micellar Solubilization of Drugs," Journal of Pharmacy \& Pharmaceutical Sciences, vol. 8, pp. 147-163, 2005.

[6] E. Brewer, J. Coleman, and A. Lowman, "Emerging Technologies of Polymeric Nanoparticles in Cancer Drug Delivery," Journal of Nanomaterials, 2011, pp. 1-10.

[7] O. Khomenko, O. Budishevska, A. Voronov, O. Kudina, I. Tarnavchyk, and S. Voronov, "Amphiphilic Oligomers Based on Diesters of Pyromellitic Acid for the Solubilization of Lipophilic Agents," International Journal of Theoretical and Applied Nanotechnology, vol. 1, pp. 17-25, 2012.

[8] G. Bao, S. Mitragotri, and S. Tong, "). Multifunctional Nanoparticles for Drug Delivery and Molecular Imaging," Annual Review of Biomedical Engineering, vol. 15, pp. 253-282, 2013. 
[9] Y.A. Dyadin and L.S. Aladko, "Clathrate Hydrates of Long-chain Tetrabutylammonium Carboxylates," Mendeleev Communications, vol. 5, pp. 239-240, 1995.

[10] L.S. Aladko and Y.A. Dyadin, "Clathrate Formation in the Tetrabutylammonium Laurate-Water System," Russian Journal of General Chemistry, vol. 69, pp. 1872-1874, 1999.

[11] R. Zana, "Partial Phase Behavior and Micellar Properties of Tetrabutylammonium Salts of Fatty Acids: Unusual Solubility in Water and Formation of Unexpectedly Small Micelles," Langmuir, vol.20, pp. 5666-5668, 2004.

[12] R. Zana, J. Schmidt, and Y. Talmon, "Tetrabutylammonium Alkyl Carboxylate Surfactants in Aqueous Solution: Self-Association Behavior, Solution Nanostructure, and Comparison with Tetrabutylammonium Alkyl Sulfate Surfactants," Langmuir, vol. 21, pp. 11658-11636, 2005.

[13] M. Summers and J. Eastoe, "Applications of Polymerizable Surfactants," Advances in Colloid and Interface Science, vol. 100, pp. 137-152, 2003.

[14] C.E. Larrabee, Jr. and E.D. Sprague, "RadiationInduced Polymerization of Sodium 10Undecenoate in Aqueous Micelle Solutions," Journal of Polymer Science Part C Polymer Letters, vol. 17, pp. 749-757, 1979.

[15] J.M. Denton, D.C. Duecker, and E.D. Sprague, "Size and Solution Behavior of Sodium 10undecenoate Oligomers," Journal of Physical Chemistry, vol. 97, pp. 756-762, 1993.

[16] T.V. Rodionova, A.Y. Manakov, Y.G. Stenin, G.V. Villevald, and T.D. Karpova, "The Heats of Fusion of Tetrabutylammonium Fluoride Ionic Clathrate Hydrates," Journal of Inclusion Phenomena and Macrocyclic Chemistry, vol. 61, pp. 107-111, 2008.

[17] H. Preston-Thomas, "The International Temperature Scale of 1990 (ITS-90)," Metrologia, vol. 27, pp. 3-10, 1990.

[18] E.D. Sprague, D.C. Duecker, and C.E. Larrabee, Jr., "The Effect of a Terminal Double Bond on the Micellization of a Simple Ionic Surfactant," Journal of Colloid and Interface Science, vol. 92, pp. 416421, 1983.

[19] A.R. Tehrani-Bagha and K. Holmberg, "Solubilization of Hydrophobic Dyes in Surfactant Solutions," Materials, vol. 6, pp. 580-608, 2013.

[20] J. Sakamoto, S. Hashimoto, T. Sugahara, Y. Inoue, and K. Ohgaki, "Thermodynamic and Raman
Spectroscopic Studies on Hydrogen + tetra- $n$-butyl Ammonium Fluoride Semi-clathrate Hydrates," Chemical Engineering Science, vol. 63, pp 57895794, 2008.

[21] H.B. Klevens, "Structure and Aggregation in Dilute Solutions of Surface Active Agents," Journal of the American Oil Chemists' Society, vol. 30, pp 78-80, 1953.

[22] C. Wang, P. Yan, H. Xing, C. Jin, and J.-X. Xiao, "Thermodynamics of Aggregation of Ammonium/Tetraalkylammonium Perfluorooctanoates: Effect of Counterions," Journal of Chemical \& Engineering Data, vol. 55, pp 1994-1999, 2010.

[23] M. Bemrraou. B.L. Bales, and R. Zana, "Effect of the Nature of the Counterion on the Properties of Anionic Surfactants. 1. Cmc, Ionization Degree at the $\mathrm{Cmc}$ and Aggregation Number of Micelles of Sodium, Cesium, Tetramethylammonium, Tetraethylammonium, Tetrapropylammonium, and Tetrabutylammonium Dodecyl Sulfates," Journal of Physical Chemistry B, vol. 107, pp 13432-13440, 2003. 